\title{
Correction to: Characteristic MRI findings of shoulder, elbow, and wrist joints in wheelchair user
}

\author{
Masafumi Sakai ${ }^{1} \cdot$ Hirotaka Mutsuzaki $^{2} \cdot$ Yukiyo Shimizu $^{3} \cdot$ Yoshikazu Okamoto $^{1} \cdot$ Katsuhiko Yatabe $^{4} \cdot$ Ichio Muraki $^{4}$. \\ Kotaro Nakajima ${ }^{5}$
}

Published online: 23 January 2021

(C) ISS 2021

Skeletal Radiology (2021) 50:171-178

https://doi.org/10.1007/s00256-020-03545-2.

In the original manuscript, this reads:

"Masafumi Sakai ${ }^{1}$ Takahiro Mutsuzaki ${ }^{2}$ Yukiyo Shimizu ${ }^{1}$

Yoshikazu Okamoto ${ }^{1}$ Katsuhiko Yatabe $^{3}$ Ichio Muraki $^{3}$

Kotaro Nakajima ${ }^{3}$."

“1 Department of Diagnostic and Interventional Radiology, Faculty of Medicine, University of Tsukuba, 2-1-1 Amakubo, Tsukuba, Ibaraki 305-8576, Japan

${ }^{2}$ Department of Orthopedics, Ibaraki Prefectural University, 4669-2 Oaza, Inashiki, Ibaraki 300-0300, Japan

${ }^{3}$ Department of Radiology, Ibaraki Prefectural University, 4669-2 Oaza, Inashiki, Ibaraki 300-0300, Japan"

This should read:

"Masafumi Sakai ${ }^{1}$ Hirotaka Mutsuzaki ${ }^{2}$ Yukiyo Shimizu ${ }^{3}$ Yoshikazu Okamoto ${ }^{1}$ Katsuhiko Yatabe $^{4}$ Ichio Muraki $^{4}$ Kotaro Nakajima",

The online version of the original article can be found at https://oi.org/ $10.1007 / \mathrm{s} 00256-020-03545-2$

Yoshikazu Okamoto

yokamoto@md.tsukuba.ac.jp

Masafumi Sakai

k4106615@kadai.jp

Hirotaka Mutsuzaki

mutsuzaki@ipu.ac.jp

Yukiyo Shimizu

shimiyukig@gmail.com

Katsuhiko Yatabe

yatabek@ami.ipu.ac.jp

Ichio Muraki

murakii@ami.ipu.ac.jp

Kotaro Nakajima

nakajimak@ipu.ac.jp
"1Department of Diagnostic and Interventional Radiology, Faculty of Medicine, University of Tsukuba, 2-1-1 Amakubo, Tsukuba, Ibaraki 305-8576, Japan

${ }^{2}$ Department of Orthopedic Surgery, Ibaraki Prefectural University of Health Sciences, 4669-2 Ami, Inashiki-gun, Ibaraki 300-0394, Japan

${ }^{3}$ Department of Rehabilitation Medicine, Faculty of Medicine, University of Tsukuba, 1-1-1 Tennodai, Tsukuba, Ibaraki 305-8575, Japan

${ }^{4}$ Department of Radiology, Ibaraki Prefectural University of Health Sciences Hospital, 4733 Ami, Inashiki-gun, Ibaraki 300-0331, Japan

${ }^{5}$ Department of Radiological Sciences, Ibaraki Prefectural University of Health Sciences, 4669-2 Ami, Inashiki-gun, Ibaraki 300-0394, Japan"

Publisher's note Springer Nature remains neutral with regard to jurisdictional claims in published maps and institutional affiliations.

1 Department of Diagnostic and Interventional Radiology, Faculty of Medicine, University of Tsukuba, 2-1-1 Amakubo, Tsukuba, Ibaraki 305-8576, Japan

2 Department of Orthopedic Surgery, Ibaraki Prefectural University of Health Sciences, 4669-2 Ami, Inashiki-gun, Ibaraki 300-0394, Japan

3 Department of Rehabilitation Medicine, Faculty of Medicine, University of Tsukuba, 1-1-1 Tennodai, Tsukuba, Ibaraki 305-8575, Japan

4 Department of Radiology, Ibaraki Prefectural University of Health Sciences Hospital, 4733 Ami, Inashiki-gun, Ibaraki 300-0331, Japan

5 Department of Radiological Sciences, Ibaraki Prefectural University of Health Sciences, 4669-2 Ami, Inashiki-gun, Ibaraki 300-0394, Japan 